\title{
Cancer Research Strategies and Cancer Care
}

\section{Tatjana Abaffy}

Department of Molecular and Cellular Pharmacology, University of Miami, Miller School of Medicine, Miami, Florida, USA

Imatinib mesylate (Gleevec) is a tyrosine kinase inhibitor currently used for treatment of BCR-ABL (Breakpoint Cluster Region-vabl ABelson murine Leukemia viral oncogene) Tyrosine Kinase (TK) positive leukemia, as well as GastroIntestinal Stromal Tumors (GIST). Imatinib represents one of the rare successful stories in drug development [1]. Imatinib binds to the catalytic site of the kinase and traps it in an inactive conformation. This success was made possible through decades of intensive and collaborative research which led to discovery of the involvement of this protein kinase in tumor pathology. However, this success though impressive, was not immortal. A resistance to the drug developed. Drug resistance is often associated with chronic treatment with anticancer drugs and is likely due to the general genomic instability well documented in cancer. It has been shown that Activation Induced Cytidine Deaminase, AICD, the enzyme that converts cytidine to uridine, causing DNA breaks and hypermutations, also causes mutations in BCR-ABL TK resulting in Imatinib resistance. The recently revealed complexity of polyclonal resistance in patients with imatinib-resistant GIST, suggests that a single next generation drug is unlikely to inhibit all mutant clones in a given patient [2].

An article by Loeb [3] gives a comprehensive perspective on this topic: "Human genome is dynamic. It is estimated that each cell undergoes $>20,000$ DNA damaging events and $>10,000$ replication errors per cell per day." He also discussed the results from the Cancer Genome Atlas, which: "...revealed that each tumor is unique and contains tens to hundreds of thousands of mutations." We also know that there is not a set of mutated genes that are diagnostic of a specific tumor. To further cite Loeb [3] "It is estimated that each cancer cell within most tumors contains $>10,000$ mutations. By the time a tumor is clinically detected it is likely to contain 108-109 cells and could, in principle, harbor $>1012$ different mutations.....Every tumor could contain mutant genes that will render some cells resistant to any single chemotherapeutic agent". Thus, the idea to target a particular gene mutation as a therapeutic strategy in most cancer cases will not work over the long-term to eliminate or even arrest cancer in the patient. In addition to this pessimistic prediction, the results from Genome-Wide Association Studies (GWAS) of Single Nucleotide Polymorphism (SNP) trait associations of common genetic variants with $>80$ diseases, showed very little predictive power and very little clinical utility $[4,5]$. These are bad news for the proponents of personalized or "tailored" medicine approach. The good news is that new approaches, like polypharmacology, the simultaneous inhibition of multiple targets, are underway [2].

In contrast to the difficult task of controlling an established tumor, the importance of identifying genetic mutations as a preventive and/or early surgical intervention strategy in people at high risk for developing cancer cannot be denied. For a small subset of cancers caused by germline mutations, molecular genetic testing to identify individuals from affected families that may carry these mutations is an imperative. For example, individuals with a germline mutation in BRCA1 or BRCA2 tumor suppressor gene are predisposed to develop breast, ovarian, prostate, pancreatic cancer or melanoma. For these individuals, regular screening using all available tests to detect the earliest stages of the disease is a must.
Implications of mutations in Krebs cycle's enzymes have shifted focus from genes to metabolism. Mutations in oncogenes and tumor suppressor genes cause alterations in signaling pathways changing tumor metabolism [6]. It has been shown that glycolysis can be regulated at many different levels; via the PI3K/Akt signaling pathway, via the c-Myc gene, via activation of HIF-1 $\alpha$ and via p53 inactivation. Thus, a metabolic pathway can be regulated by many different signaling pathways and at many different levels reflecting both its significance and its tight control. The existence of the cross-talk between different signaling pathways became apparent while studying molecular mechanisms of drug resistance. Recently it was demonstrated that melanoma cells evoke resistance to BRAF inhibitor by switching to IGF-1R/PI3K signaling pathway which promotes cell survival and growth and at the same time causes drug resistance [7]. In addition, we should not forget about the feedback loop activation constantly looming inside the cell. As an example, a prolonged treatment with mTOR inhibitors, in particular rapamycin, induces negative feedback regulation of PI3K/Akt signaling that contributes to the resistance of cancer cells [8]. Thus, a better understanding not of a single discrete pathway, but of the complex molecular network using a "systems" approach is an imperative.

Non-targeted antineoplastic drugs like antimetabolites and alkylating agents (methotrexate, 5-fluorouracil, cisplatin and vinca alkaloids) developed in the 1960s, long before targeted genetic drugs, have been used with no less success in treating many cancers [9]. This poses a question: A targeted or a non-targeted therapeutic approach? Do we have the luxury of abandoning any of these approaches? As stated by Evans et al. [5]:"The nature of scientific progress is arguably not optimized by a rigid allocation of resources to purely practical need."

We are all intensely aware of the spiraling cost of cancer care. So far, the global funding for genomics was estimated to be almost $\$ 3$ billion per year, calculated for the period 2003-2006 [10]. The estimated total cost of cancer care in the United States in 2020 is expected to be $\$ 158$ billion assuming the most recent observed patterns of incidence, survival, and costs remain the same [11]. The following are examples of cancer treatment costs: Fluorouracil treatment for metastatic colorectal cancer cost about $\$ 70$ and median survival of cancer patients is about 12 months. New FDA-approved FOLFOX regimen consisting of fluorouracil, irinotecan, and oxaliplatin costs $\$ 11,889$ and extends the median survival to 21 months [12]. The annual estimated cost of treating melanoma in the US is over $\$ 3.1$ billion, with $90 \%$ of treatment costs

*Corresponding author: Tatjana Abaffy, Department of Molecular and Cellula Pharmacology, University of Miami, Miller School of Medicine, Miami, Florida, USA Tel: (305) 322-0115; E-mail: TAbaffy@med.miami.edu

Received September 07, 2011; Accepted September 10, 2011; Published September 14, 2011

Citation: Abaffy T (2011) Cancer Research Strategies and Cancer Care. J Pharmacogenom Pharmacoproteomics 2:e103. doi:10.4172/2153-0645.1000e103

Copyright: @ 2011 Abaffy T. This is an open-access article distributed under the terms of the Creative Commons Attribution License, which permits unrestricted use, distribution, and reproduction in any medium, provided the original author and source are credited. 
associated with therapy for advanced disease [13]. The FDA-approved treatments of metastatic melanoma are: high dose of interleukin-2 (IL-2), ipilimumab (anti-CTLA4 antibody), peginterferon alfa-2b and vemurafenib (inhibitor of the BRAFV600E gene). The median survival increment due to these treatments is measured in months. In contrast, Adoptive Cell Transfer (ACT) therapy, a direct transfer of activated $\mathrm{T}$ cells in lympho-depleted hosts, demonstrated disappearance of all melanoma tumor in 20 of 93 patients treated. $95 \%$ of these patients have long-lasting, disease-free complete response for more than 7 years [14-16]. These encouraging results should receive more widespread attention and support; and not just from the scientific community. The cost of ACT based immunotherapy is approximately $\$ 20,000$ plus costs associated with hospitalization for treatment. Weber et al estimated that this is less than a conventional treatment with targeted small molecules or antibody, not to mention durable long term remission of disease, and in some cases complete cure [17].

The importance of early screening tests and cancer detection technologies cannot be overstated. We know that screening and early detection is the best way to fight cancer. Recently, the research from the National Lung Screening Trial (NLST) showed a 20\% reduction in DEATHS from lung cancer among current or former heavy smokers who were screened with low-dose helical computed tomography (CT) versus those screened by $\mathrm{x}$-rays [18].

In order to improve cancer care, to reduce morbidity and mortality, all of the above mentioned strategies should be pursued by scientific community, clinicians, health professionals and legislators. In addition, every one of us must adopt cancer prevention strategies, including healthy lifestyle and regular exercise. Regular cancer screening, especially for those at risk, must become the standard of care. Early detection enables the most effective anti-cancer strategies.

\section{References}

1. Druker BJ, Tamura S, Buchdunger E, Ohno S, Segal GM, et al. (1996) Effects of a selective inhibitor of the Abl tyrosine kinase on the growth of Bcr-Abl positive cells. Nat Med 2: 561-566.

2. Taylor BS, Barretina J, Maki RG, Antonescu CR, Singer S, et al. (2011) Advances in sarcoma genomics and new therapeutic targets. Nat Rev Cancer 11: $541-557$

3. Loeb LA (2011) Human cancers express mutator phenotypes: origin, consequences and targeting. Nat Rev Cancer 11: 450-457.

4. Hindorff LA, Sethupathy P, Junkins HA, Ramos EM, Mehta JP, et al. (2009) Potential etiologic and functional implications of genome-wide association loci for human diseases and traits. Proc Natl Acad Sci U S A 106: 9362-9367.

5. Evans JP, Meslin EM, Marteau TM, Caulfield T (2011) Genomics. Deflating the genomic bubble. Science 331: 861-862.

6. Cairns RA, Harris IS, Mak TW (2011) Regulation of cancer cell metabolism. Nat Rev Cancer 11: 85-95.

7. Villanueva J, Vultur A, Lee JT, Somasundaram R, Fukunaga-Kalabis M, et al. (2010) Acquired resistance to BRAF inhibitors mediated by a RAF kinase switch in melanoma can be overcome by cotargeting MEK and IGF-1R/PI3K. Cancer Cell 18: 683-695.

8. Wan X, Harkavy B, Shen N, Grohar P, Helman LJ (2007) Rapamycin induces feedback activation of Akt signaling through an IGF-1R-dependent mechanism. Oncogene 26: 1932-1940.

9. Arbiser JL, Fisher DE (2011) Fisetin: a natural fist against melanoma? J Invest Dermatol 131: 1187-1189.

10. Pohlhaus JR, Cook-Deegan RM (2008) Genomics research: world survey of public funding. BMC Genomics 9: 472 .
11. Mariotto AB, Yabroff KR, Shao Y, Feuer EJ, Brown ML (2011) Projections of the cost of cancer care in the United States: 2010-2020. J Natl Cancer Inst 103: 117-128.

12. Schrag D (2004) The price tag on progress--chemotherapy for colorecta cancer. N Engl J Med 351: 317-319.

13. Wich LG, Hamilton HK, Shapiro RL, Pavlick A, Berman RS, et al. (2009) Developing a multidisciplinary prospective melanoma biospecimen repository to advance translational research. Am J Transl Res 1: 35-43.

14. Dudley ME, Yang JC, Sherry R, Hughes MS, Royal R, et al. (2008) Adoptive cell therapy for patients with metastatic melanoma: evaluation of intensive myeloablative chemoradiation preparative regimens. J Clin Oncol 26: 52335239 .

15. Rosenberg SA, Yang JC, Sherry RM, Kammula US, Hughes MS, et al. (2011) Durable complete responses in heavily pretreated patients with metastatic melanoma using T-cell transfer immunotherapy. Clin Cancer Res 17: 45504557.

16. Dudley ME (2011) Adoptive cell therapy for patients with melanoma. J Cancer 2: 360-362.

17. Weber J, Atkins M, Hwu P, Radvanyi L, Sznol M, et al. (2011) White paper on adoptive cell therapy for cancer with tumor-infiltrating lymphocytes: a report of the CTEP subcommittee on adoptive cell therapy. Clin Cancer Res 17: 16641673.

18. Aberle DR, Adams AM, Berg CD, Black WC, Clapp JD, et al. (2011) Reduced Lung-Cancer Mortality with Low-Dose Computed Tomographic Screening. N Engl J Med 365: 395-409. 